TITLE:

\title{
Wave propagation in a weak gravitational field and the validity of the thin lens approximation
}

\section{$\operatorname{AUTHOR(S):~}$}

Suyama, T; Takahashi, R; Michikoshi, S

\section{CITATION:}

Suyama, T ... [et al]. Wave propagation in a weak gravitational field and the validity of the thin lens approximation. PHYSICAL REVIEW D 2005, 72(4): 043001.

ISSUE DATE:

2005-08

URL:

http://hdl.handle.net/2433/49932

RIGHT:

Copyright 2005 American Physical Society 


\title{
Wave propagation in a weak gravitational field and the validity of the thin lens approximation
}

\author{
Teruaki Suyama, ${ }^{1}$ Ryuichi Takahashi, ${ }^{2}$ and Shugo Michikoshi ${ }^{1}$ \\ ${ }^{1}$ Department of Physics, Kyoto University, Kyoto 606-8502, Japan \\ ${ }^{2}$ Division of Theoretical Astrophysics, National Astronomical Observatory of Japan, Mitaka, Tokyo 181-8588, Japan
}

(Received 9 May 2005; published 11 August 2005)

\begin{abstract}
Wave effects can be important for the gravitational lensing of gravitational waves. In such a case, wave optics must be used instead of geometric optics. We consider a plane wave entering a lens object and solve numerically the wave equation for three lens models: the uniform density sphere, the singular isothermal sphere, and the Hernquist model. By comparing our numerical solutions with the analytical solutions under the thin lens approximation, we evaluate the error of this approximation. The results show that the relative error of the thin lens approximation is small if the geometrical thickness of the lens is much smaller than the distance between the lens and the observer.
\end{abstract}

DOI: 10.1103/PhysRevD.72.043001

\section{INTRODUCTION}

Gravitational waves from coalescing compact binaries composed of neutron stars or black holes are the most promising targets for ground-based as well as space-based detectors. By applying the matched filtering technique which uses our theoretical predictions of wave forms obtained by the post-Newtonian computations, we can extract the binary parameters such as the masses of each compact object, distance to the source, spatial positions of the source, and so on [1].

One possibility which alters the predicted wave forms calculated with high precision is the gravitational lensing of gravitational waves. If a massive object lies suitably between the source and the observer, gravitational lensing of gravitational waves occurs. One important point is that since the wavelength of gravitational waves we are interested in is much larger than that of light, a situation where the geometrical optics approximation breaks down can be realized in some cases. As is discussed by many authors [2-6], if the wavelength is larger than the Schwarzschild radius of the lens object, the diffraction effect becomes important and the magnification approaches to unity. Therefore we must use wave optics rather than the geometric optics for

$$
M_{L} \lesssim 10^{8} M_{\odot}\left(\frac{f}{\mathrm{mHz}}\right)^{-1},
$$

where $M_{L}$ is a mass of the lens and $f$ is the frequency of the gravitational waves. This frequency $(\mathrm{mHz})$ is the case for the planned detector laser interferometer space antenna (LISA) [7].

Further because the gravitational waves from a compact binary are coherent, interference between lensed waves is important. Note that this situation is not in general realized in the case of gravitational lensing of electromagnetic wave such as visible light. Since light is emitted from microscopic region (usually atomic size) which is much smaller than the size of the source, each phase of the
PACS numbers: 95.30.Sf, 04.30.Nk, 42.25.Hz, 98.62.Sb

electromagnetic wave emitted from different points has no correlation and thus interference effect vanishes.

If we assume the coalescence of super massive black holes (SMBHs) of mass $10^{4} \sim 10^{7} M_{\odot}$ as the source of the gravitational waves, it can be detected even if the sources are located at the cosmological distance $(z>5)$. Event rate of SMBH-SMBH merger for LISA is estimated as $0.1 \sim$ $10^{2}$ event/yr [8] and lensing probability becomes several percent. Hence, some lensing events per year will be detected by LISA.

Motivated by the fact that wave effects can be detected for the gravitational lensing of gravitational waves, there are now growing interests in the wave optics in gravitational lensing [9-16]. However we have to solve wave equation which is generally partial differential equation between the source and the observer in order to obtain the lensed wave form at the observer. Except for a few special cases, exact solutions of wave equation are not known at present. Several authors have used thin lens approximation which reduces wave equation to double integral for single lens object (for multilens objects, integration becomes multi-integral, see [14]) and thus makes the problem easier $[15,17]$. In geometric optics the trajectory of light ray is obtained by solving geodesic equation, and it is known that thin lens approximation is valid [17]. However there have been no studies or comments about the validity of the thin lens approximation in the framework of wave optics.

In this paper, we develop a formulation to solve the wave equation for a spherically symmetric lens, where a partial differential equation reduces to a set of ordinary differential equations. We also solve those equations for simple lens models: the uniform density sphere, the singular isothermal sphere, and the Hernquist model and evaluate the error of the thin lens approximation.

This paper is organized as follows. In Section II, we briefly review wave optics in gravitational lensing under the thin lens approximation. In Section III, we develop a formulation to solve the scattering problem of gravitational 
waves by a lens. In Section IV, we present our numerical results and discuss the validity of the thin lens approximation. Section V is a summary. We use unit of $c=1$.

\section{GRAVITATIONALLY LENSED WAVEFORM UNDER THE THIN LENS APPROXIMATION}

We consider the wave propagation under the gravitational fields of a lens. We assume that the spacetime metric is a Minkowski spacetime plus a small perturbation due to the existence of a static lensing object. Then the metric can be written as

$$
d s^{2}=g_{\mu \nu} d x^{\mu} d x^{\nu}=-(1+2 U) d t^{2}+(1-2 U) d \vec{x}^{2},
$$

where $U$ is a Newtonian potential of the lensing object. We consider a propagation of scalar waves $\phi$, instead of gravitational waves, since the wave equation for $\phi$ is the same as that for gravitational waves [18]. The scalar wave equation, $\partial_{\mu}\left(\sqrt{-g} g^{\mu \nu} \partial_{\nu} \phi\right)=0$, with the metric (2) is rewritten as

$$
\left(\triangle+\omega^{2}\right) \phi(\vec{x})=4 \omega^{2} U(\vec{x}) \phi(\vec{x}),
$$

where we assume that the wave is monochromatic with the

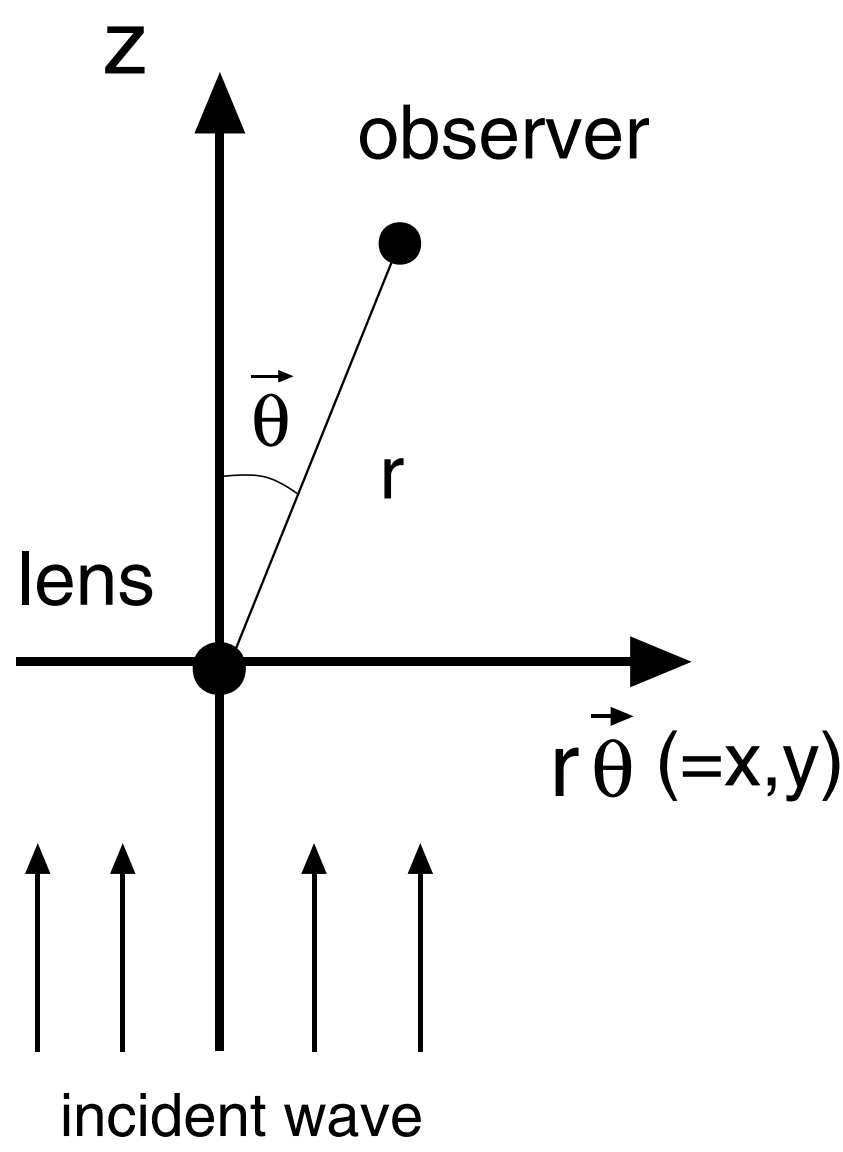

FIG. 1. Lensing configuration. The lens is the origin of coordinate axes, while the observer position is $(r, \vec{\theta})$ with $\theta \ll 1$. The incident wave is a plane wave propagating in the z-direction. angular frequency $\omega$. The above equation was solved by using the Kirchhoff diffraction integral (see [17], Sec. 4.7 and 7) under the thin lens approximation.

We show lensing configuration in Fig. 1. We choose a position of the lens as the origin of the polar coordinate system $(r, \theta, \phi)$. The observer position is $r$ and $\vec{\theta}=$ $(\theta \cos \phi, \theta \sin \phi)$ with $\theta \ll 1$. The incident wave is a plane wave propagating in the z-direction. Denoting the incident wave as $\phi^{0}$, we have $\phi^{0}=e^{i \omega r \cos \theta}$.

In this section, we assume the thin lens approximation, in which the wave is scattered only on the thin lens plane at $z=0$, and the lens is characterized by the surface mass density $\Sigma(\vec{s})$, where $\vec{s}=(x, y)$. The two-dimensional potential $\psi(\vec{s})$ in $z=0$ plane is defined as

$$
\psi(\vec{s})=2 \int_{-\infty}^{\infty} d z U(\vec{s}, z) .
$$

Here, $\psi$ is also obtained from the surface density by using $\nabla_{s}^{2} \psi(\vec{s})=8 \pi \Sigma(\vec{s})$.

It is useful to define the amplification factor $F$ (which is called the transmission factor in Ref. [17]) as $F=\phi / \phi_{o}$, where $\phi$ is the gravitationally lensed waveform obtained by solving Eq. (3) and $\phi^{0}$ is the incident wave. The amplification factor at the observer under the thin lens approximation is given by [17]

$$
F_{\text {thin }}(r, \vec{\theta})=\frac{\omega}{2 \pi i r} \int d^{2} s e^{i(\omega / 2 r)|r \vec{\theta}-\vec{s}|^{2}-i \omega \psi(\vec{s})} .
$$

Here, $F_{\text {thin }}$ is normalized so that $F_{\text {thin }}=1$ for $\psi=0$.

\section{FORMULATION OF NUMERICAL CALCULATION}

In this section, we develop a formulation to solve the scattering problem of gravitational waves by lensing object which is applicable to the case where the lens potential is spherically symmetric. A situation we will consider is that plane wave is entering weak gravitational field which is spherically symmetric. When the lensing object is spherically symmetric, a scattering problem can be reduced to a problem of determining so-called phase shift which is used to probe the nature of nuclear physics and is also useful for the scattering by black holes (BHs) where the assumption of weak gravitational field breaks down [19].

We have to solve Eq. (3) in order to evaluate quantities such as the amplification factor which can be compared with the one derived under the thin lens approximation. To solve Eq. (3), we choose a center of the lens as the origin of coordinate system. Then $U$ becomes a function which depends only on the radius coordinate $r$. Now let us expand $\phi$ in terms of Legendre function

$$
\phi(\xi, \theta)=\sum_{\ell=0}^{\infty} \frac{g_{\ell}(\xi)}{\xi} P_{\ell}(\cos \theta),
$$

where $\xi$ is a dimensionless variable defined by $\xi \equiv r \omega$. 
Then equations for $g_{\ell}(\xi)$ are

$$
\left(\frac{d^{2}}{d \xi^{2}}+1-4 \tilde{U}(\xi)-\frac{\ell(\ell+1)}{\xi^{2}}\right) g_{\ell}(\xi)=0,
$$

where we have used $\tilde{U}$ instead of $U$ in order to stress that $\tilde{U}$ is a function of $\xi$.

For a point mass lens, i.e. $\tilde{U}(\xi)=-p /(2 \xi)$, where $p \equiv$ $2 G M \omega$ and $M$ is a lens mass, analytic solutions of Eq. (7) are known as Coulomb wave functions [20]. The solution which is regular at $\xi=0$ is

$$
\begin{aligned}
F_{\ell}(-p, \xi)= & e^{\frac{\pi}{2} p} \frac{\Gamma(\ell+1-i p)}{\Gamma(2(\ell+1))} 2^{\ell} \xi^{\ell+1} e^{i \xi+i \sigma_{\ell}} \\
& \times F(\ell+1-i p ; 2(\ell+1) ;-2 i \xi), \\
\sigma_{\ell} \equiv & \arg \Gamma(\ell+1+i p),
\end{aligned}
$$

where $F$ is the confluent hypergeometric function. The solution which is singular at $\xi=0$ is

$$
\begin{aligned}
G_{\ell}(-p, \xi)= & \xi^{\ell+1} 2^{\ell} e^{-(\pi / 2) p}(-i)^{2 \ell+1} e^{i \xi-i \sigma_{\ell}} \\
& \times U(\ell+1-i p ; 2(\ell+1) ;-2 i \xi)+\text { c.c., }
\end{aligned}
$$

where $U$ is defined as

$$
U(a ; b ; z)=\frac{1}{\Gamma(a)} \int_{0}^{\infty} d t e^{-z t} t^{a-1}(1+t)^{b-a-1} .
$$

The asymptotic form of these functions are

$$
\begin{aligned}
& F_{\ell}(-p, \xi) \underset{\xi \gg 1}{\longrightarrow} \sin \left(\xi+p \log 2 \xi-\frac{\pi}{2} \ell-\sigma_{\ell}\right), \\
& G_{\ell}(-p, \xi) \underset{\xi \gg 1}{\longrightarrow} \cos \left(\xi+p \log 2 \xi-\frac{\pi}{2} \ell-\sigma_{\ell}\right) .
\end{aligned}
$$

The term $p \log 2 \xi$ in the phase of trigonometric functions represents the nature of long range force which is characteristic of Coulomb force.

On the contrary, a solution of Eq. (3) that a plane wave is entering a point mass lens is well known and is given by [21]

$$
\phi_{p}=e^{(\pi / 2) p} \Gamma(1-i p) e^{i \xi \cos \theta} F(i p ; 1 ; i \xi(1-\cos \theta)) .
$$

Because the solution Eq. (12) is regular at $\xi=0$, it is written as a partial wave sum of regular Coulomb wave function,

$$
\begin{aligned}
\phi_{p} & =\sum_{\ell=0}^{\infty} a_{\ell} \frac{F_{\ell}(-p, \xi)}{\xi} P_{\ell}(\cos \theta), \\
a_{\ell} & =i^{\ell}(2 \ell+1) e^{-i \sigma_{\ell}} .
\end{aligned}
$$

For an extended lensing object, analytic solution of Eq. (3) does not exist usually. However if the lensing object exists only in a finite region, then the solution of Eq. (3) outside the lens can be written as a summation of partial waves which are now a linear combination of two inde- pendent Coulomb wave functions. By determining a coefficient of each Coulomb wave function, we can calculate the wave form $\phi$ outside the lens. In the aim of only determining the wave form far from the lensing object, we do not need to know the expression of $\phi$ inside the lens.

Now let us write the solution of Eq. (3) as

$$
\phi=\phi_{p}+\phi_{s} \text {. }
$$

Thus $\phi_{s}$ represents the scattered wave which arises due to the deviation of lens from a point mass. There may be no incoming scattered wave to the lens from infinity, so we assume the asymptotic form of $\phi_{s}$ as

$$
\phi_{s}(\xi, \theta)=\sum_{\ell=0}^{\infty} \frac{e^{i \xi+i p \log 2 \xi-i \frac{\pi}{2} \ell-i \sigma_{\ell}}}{2 i \xi} s_{\ell} P_{\ell}(\cos \theta),
$$

where $s_{\ell}$ are undetermined complex numbers, but not arbitrary. In order that $\phi$ in Eq. (14) satisfy the wave equation (3), $s_{\ell}$ must be related to $a_{\ell}$ as

$$
s_{\ell}=a_{\ell}\left(e^{2 i \delta_{\ell}}-1\right),
$$

where $\delta_{\ell}$ are real numbers. In terms of $\delta_{\ell}, \phi$ is written as

$$
\begin{aligned}
\phi(\xi, \theta)= & \sum_{\ell=0}^{\infty} a_{\ell} e^{i \delta_{\ell}}\left(\cos \delta_{\ell} \frac{F_{\ell}(-p, \xi)}{\xi}\right. \\
& \left.+\sin \delta_{\ell} \frac{G_{\ell}(-p, \xi)}{\xi}\right) P_{\ell}(\cos \theta) .
\end{aligned}
$$

Thus we can calculate wave form outside the lens object by determining the phase shift $\delta_{\ell}$. Here, $\delta_{\ell}$ are determined by matching a solution of Eq. (7) with Eq. (17) at a radius $\xi_{0}$ being outside the lens. Then, we have

$$
\tan \delta_{\ell}=\frac{g_{\ell}\left(\xi_{o}\right) F_{\ell}^{\prime}\left(-p, \xi_{o}\right)-g_{\ell}^{\prime}\left(\xi_{o}\right) F_{\ell}\left(-p, \xi_{o}\right)}{g_{\ell}^{\prime}\left(\xi_{o}\right) G_{\ell}\left(-p, \xi_{o}\right)-g_{\ell}\left(\xi_{o}\right) G_{\ell}^{\prime}\left(-p, \xi_{o}\right)},
$$

where $g_{\ell}$ is a solution of Eq. (7) which is to be calculated numerically. We set the initial condition of Eq. (7) at $\xi=0$ is that $g_{\ell}$ is regular. The range of $\delta_{\ell}$ is from $-\pi / 2$ to $\pi / 2$.

\section{RESULTS}

We investigate the validity of the thin lens approximation for three lens models; the uniform density sphere, the singular isothermal sphere, and the Hernquist model.

\section{A. Uniform density sphere}

We first present the results for uniform density sphere which has the simplest structure next to the point mass. The gravitational potential for the uniform density sphere is given by

$$
U(r)=\left\{\begin{array}{cc}
-\frac{G M}{2 R}\left(3-\frac{r^{2}}{R^{2}}\right) & (\mathrm{r} \leq \mathrm{R}) \\
-\frac{G M}{r} & (\mathrm{r} \geq \mathrm{R}),
\end{array}\right.
$$

where $r$ is the distance from the center of the sphere, $R$ is 
the radius of the sphere, and $M$ is the lens mass. Here we consider the case that $R$ is larger than the Einstein radius $r_{E}$, in which case the effect of the size of the lens object is expected to be important. For $R<r_{E}$, the result is almost the same as for point lens mass and it is known that amplitude of the amplification factor for the point mass lens coincides with that in the thin lens approximation [15].
The amplification factor in the thin lens approximation Eq. (5) for the case of uniform density sphere is written as

$$
F_{\text {thin }}(r, \theta)=-i \frac{\omega}{r} e^{(i / 2) \omega r \theta^{2}} \int_{0}^{\infty} d s s J_{0}(\omega s \theta) e^{i w\left(\left(s^{2} / r\right)-\psi(s)\right)},
$$

where $J_{0}$ is the 0 th Bessel function and $\psi$ is given by

$$
\psi(s)=\left\{\begin{array}{cc}
4 M \log \left[1+\sqrt{1-(s / R)^{2}}\right]-\frac{4 M}{3}\left(4-(s / R)^{2}\right) \sqrt{1-(s / R)^{2}} & (\mathrm{~s} \leq \mathrm{R}) \\
4 M \log (s / R) & (\mathrm{s} \geq \mathrm{R}) .
\end{array}\right.
$$

Next let us calculate $F$ numerically by using the method developed in the previous section. We first show $\delta_{\ell}$ as a function of $\ell$ in Fig. 2. We see that $\delta_{\ell}$ decreases as $\ell$ increases. In particular, above around $\ell=90, \delta_{\ell}$ rapidly approaches zero. This is because the $\ell$ th partial wave can be interpreted as an incident particle with impact parameter $\ell / \omega$. Partial waves of $\ell \geqslant \omega R$ pass through the lens potential outside the lens object and the gravitational effects on these partial waves are the same as point mass, which implies $\delta_{\ell}$ becomes zero.

Figure 3 shows the error of the amplification factor for the uniform density sphere as a function of lens parameters $\omega R, \omega r, G M \omega$, and $\theta$, respectively. The vertical axis is the error $\Delta$ defined as, $\Delta^{2} \equiv\left(|F|-\left|F_{\text {thin }}\right|\right)^{2} /\left|F_{\text {thin }}\right|^{2}$. The normalized Einstein radius is $\omega r_{E}=2 \omega \sqrt{G \overline{M r}}=$ $200(G M \omega / 1)^{1 / 2}\left(\omega r / 10^{4}\right)^{1 / 2}$. The radius of sphere $\omega R(=$ $100)$ is comparable to the Einstein radius.

The top left and right panels show $\Delta$ as a function of $\omega R$ and $\omega r$, respectively. We find that averaged in $R$ or $r$ over a

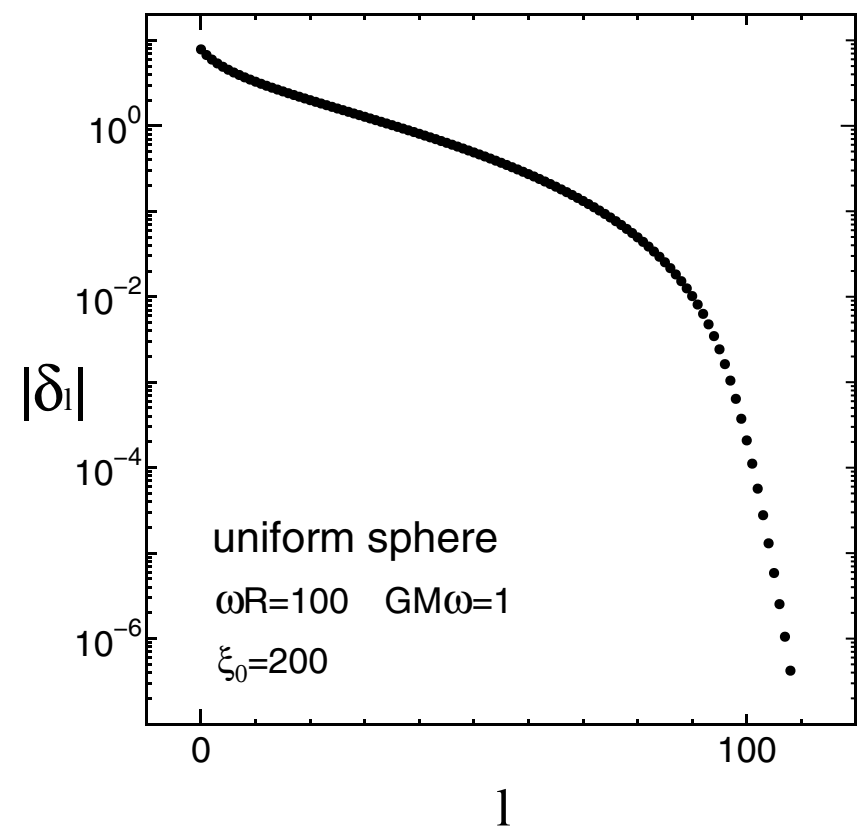

FIG. 2. $\delta_{\ell}$ as a function of $\ell$ for the uniform density sphere. period of oscillation, $\Delta$ is proportional to $R / r$. Also we find that $\Delta$ remains smaller than $R / r$ which is much smaller than unity. (The dashed lines denote the ratio of the radius to distance $R / r$.) This suggests that geometrical thickness of lens $R / r$ is a suitable measure of the validity of thin lens approximation in wave optics.

The bottom left panel shows dependence of $\Delta$ on $G M \omega$. We find that $\Delta$ has a peak around $G M \omega=10$ and decreases as $G M \omega$ increases for $G M \omega>10$. This result shows that the thin lens approximation is valid for the wavelength where we can use geometric optics instead of wave optics. The reason why thin lens approximation becomes valid in the geometric optics is that the deflection angle can be evaluated by using the thin lens potential if the distance between lens and observer is much larger than the lens size (see Ref. [17], p. 124). For this panel too, $\Delta$ remains smaller than $R / r$ in all range of frequencies of calculation.

The bottom right panel shows dependence of $\Delta$ on $\theta$. We find that $\Delta$ becomes maximum at close to $\theta=0$ and decreases as $\theta$ becomes larger. This is because for large $\theta$ such that $r \theta \gtrsim R(\theta \gtrsim 0.01$ for a case of Fig. 3), the size effect of lens becomes negligible and the lensed waveform becomes the same as for the point mass lens for which it is known that thin lens approximation is valid. Except for a small region of $\theta$ around where $\Delta$ takes maximum value, $\Delta$ is smaller than $R / r$.

To summarize, for the uniform density sphere, the relative error of the amplification factor in the thin lens approximation is suppressed within the ratio of its radius to distance $R / r$, which is much smaller than unity in a realistic astrophysical situation and is the largest for $\lambda \sim G M$ ( $\lambda$ is the wavelength).

\section{B. Singular Isothermal Sphere (SIS) model}

We also calculated $\Delta$ for singular isothermal sphere which is a model of galaxies, dark matter haloes, and star clusters. The density profile of SIS model is $\rho(r)=$ $v^{2} /\left(2 \pi r^{2}\right)$ where $v$ is the velocity dispersion. For numerical calculation, we have to introduce cutoff radius $r_{c}$ because gravitational potential does not approach to $\propto$ $r^{-1}$ far from the lens without cutoff. Thus we here assume 


\section{WAVE PROPAGATION IN A WEAK GRAVITATIONAL ...}

that the density vanishes for $r>r_{c}$. Newton potential becomes

$$
U(r)=\left\{\begin{array}{cc}
\frac{G M}{r_{c}}\left(\ln \frac{r}{r_{c}}-1\right) & \left(\mathrm{r} \leq \mathrm{r}_{\mathrm{c}}\right) \\
-\frac{G M}{r} & \left(\mathrm{r} \geq \mathrm{r}_{\mathrm{c}}\right),
\end{array}\right.
$$

where $M$ is the mass inside the cutoff radius $r_{c}: M=$ $2 v^{2} r_{c}$.

$F_{\text {thin }}$ is given by Eq. (20), and $\psi(s)$ is given by

$$
\psi(s)=\left\{\begin{array}{cc}
-\frac{8 G M}{r_{c}} \sqrt{r_{c}^{2}-s^{2}}+\frac{4 G M}{r_{c}} s \arctan \frac{\sqrt{r_{c}^{2}-s^{2}}}{s} & \\
+4 G M \ln \left(1+\sqrt{1-\left(s / r_{s}\right)^{2}}\right) & \left(\mathrm{s} \leq \mathrm{r}_{\mathrm{c}}\right) \\
8 G M \ln \left(s / r_{c}\right) & \left(\mathrm{s} \geq \mathrm{r}_{\mathrm{c}}\right) .
\end{array}\right.
$$

Figure 4 presents dependence of $\Delta$ on parameters, $\omega r_{c}$, $\omega r, \omega G M$, and $\theta$. We find that the qualitative behavior is the same as the case of uniform density sphere. We see from the top left panel and the top right panel that thin lens approximation becomes worse as cutoff approaches to observer's distance $r$ from the center of SIS.
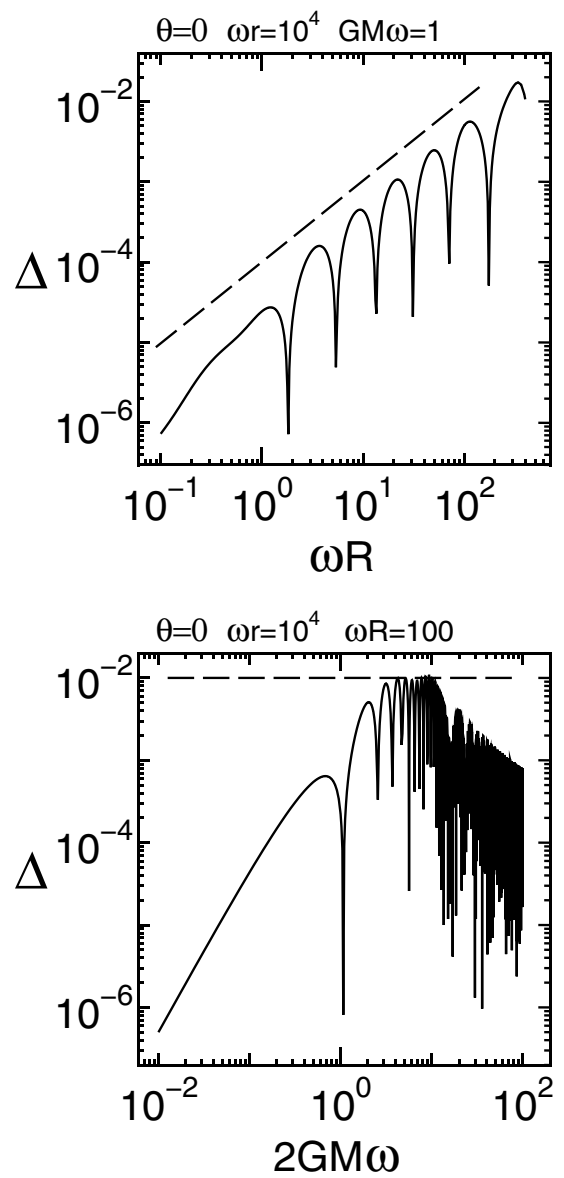

PHYSICAL REVIEW D 72, 043001 (2005)

\section{Hernquist model}

We also calculated $\Delta$ for Hernquist model [22], which fits well the luminosity distribution of many elliptical galaxies and bulges. Its density profile is given by

$$
\rho(r)=\frac{\rho_{s}}{\left(r / r_{s}\right)\left(1+r / r_{s}\right)^{3}},
$$

where $r_{s}$ is a scale length and $\rho_{s}$ is a characteristic density. For numerical calculation, we introduce cutoff radius $r_{c}$ for the same reason as SIS. For $r<r_{c}$ the density is given in Eq. (24), while for $r>r_{c}$ the density vanishes. Then, Newton potential becomes

$$
U(r)=\left\{\begin{array}{cc}
-\frac{G M}{r+r_{s}}\left(\frac{r_{s}+r_{c}}{r_{c}}\right)^{2}+\frac{r_{s}}{r_{c}} G M & \left(\mathrm{r} \leq \mathrm{r}_{\mathrm{c}}\right) \\
-\frac{G M}{r} & \left(\mathrm{r} \geq \mathrm{r}_{\mathrm{c}}\right)
\end{array}\right.
$$

where $M$ is the mass inside the cutoff radius $r_{c}: M=$ $2 \pi \rho_{s} r_{s}^{3}\left[r_{c} /\left(r_{s}+r_{c}\right)\right]^{2}$.

$F_{\text {thin }}$ is given by Eq. (20), and $\psi(s)$ is given by
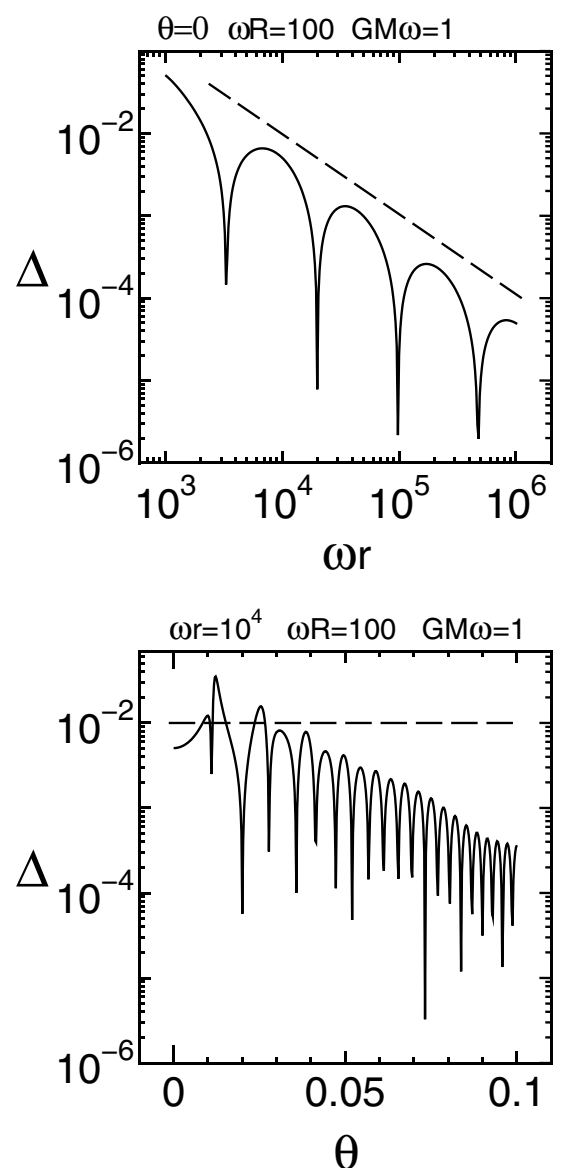

FIG. 3. Error of the amplification factor for the uniform density sphere. The horizontal axis is the radius $\omega R$ (top left), the distance $\omega r$ (top right), the mass $2 G M \omega(=p)$ (bottom left), and the angle $\theta$ (bottom right). The dashed lines denote the ratio of radius to distance $R / r$. 

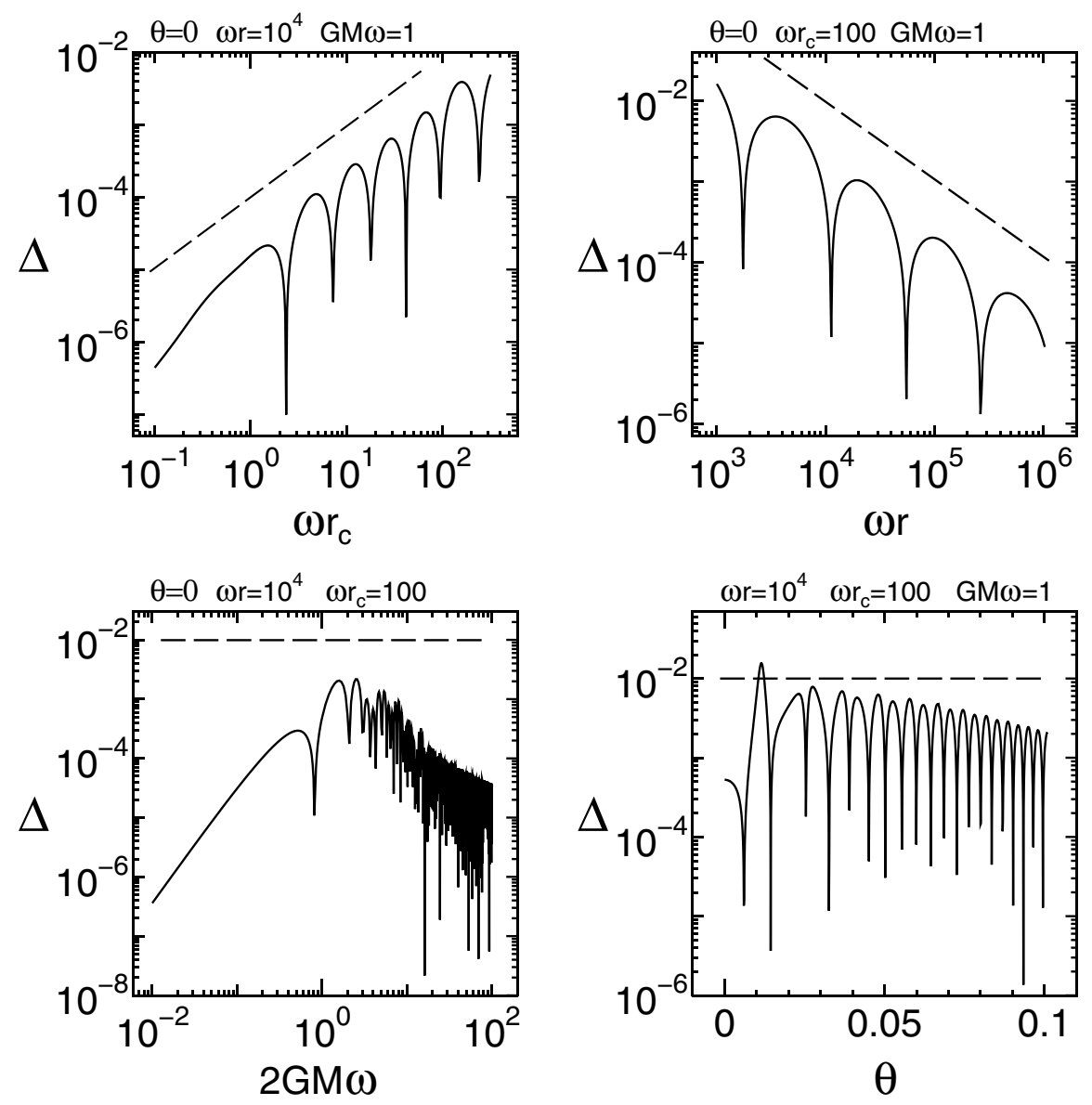

FIG. 4. Same as Fig. 3, but for the SIS model. The dashed lines denote the ratio of cutoff radius to distance $r_{c} / r$.

$$
\psi(s)=\left\{\begin{array}{cc}
-4 M \frac{r_{s}^{2}+2 r_{s} r_{c}}{r_{c}^{2}} \operatorname{arccosh} \frac{r_{s}}{s}+4 M \ln s+4 \frac{r_{s}}{r_{c}^{2}} M \sqrt{r_{s}^{2}-s^{2}}+\frac{4 M r_{s}\left(r_{s}+r_{c}\right)^{2}}{r_{c}^{2} \sqrt{r_{s}^{2}-s^{2}}} \operatorname{arctanh}\left[\frac{\sqrt{\left(r_{s}^{2}-s^{2}\right)\left(r_{c}^{2}-s^{2}\right)}}{s^{2}+r_{c} r_{s}}\right] & \left(\mathrm{s} \leq \mathrm{r}_{\mathrm{s}}\right) \\
-4 M \frac{r_{s}^{2}+2 r_{s} r_{c}}{r_{c}^{2}} \operatorname{arccosh} \frac{r_{s}}{s}+4 M \ln s+4 \frac{r_{s}}{r_{c}^{2}} M \sqrt{r_{s}^{2}-s^{2}}+\frac{4 M r_{s}\left(r_{s}+r_{c}\right)^{2}}{r_{c}^{2} \sqrt{s^{2}-r_{s}^{2}}} \arctan \left[\frac{\sqrt{\left(s^{2}-r_{s}^{2}\right)\left(r_{c}^{2}-s^{2}\right)}}{s^{2}+r_{c} r_{s}}\right] & \left(\mathrm{r}_{\mathrm{s}} \leq \mathrm{s} \leq \mathrm{r}_{\mathrm{c}}\right) \\
4 M \ln s & \left(\mathrm{r} \geq \mathrm{r}_{\mathrm{c}}\right)
\end{array}\right.
$$

Figure 5 shows $\Delta$ for Hernquist model. We see that the behavior of $\Delta$ is almost the same as SIS.

\section{SUMMARY}

In this paper we discussed the validity of the thin lens approximation in the framework of wave optics.

In Sec. III, we developed a formalism to solve the wave equation for the spherically symmetric potential. In this case, the partial differential equation can be reduced to a set of ordinary differential equations. The method we used is to determine the so-called phase shift which represents the difference of scattered waves between the point mass lens and an arbitrary spherically symmetric potential. This formalism is only applicable to the spherically symmetric lens whose size is finite. For lens models such as the SIS profile which extends to infinity, we have to introduce cutoff to make the total mass finite.
We also solved the wave equation numerically for the spherically symmetric potential. By numerical calculations, we found that the error of the thin lens approximation for the simple lens models is the same as or smaller than the geometric thickness of the lens, $s / r$, where $s$ is the size of the lens and $r$ is the distance between the lens and the observer. The error is the largest for the wavelength comparable to the Schwarzschild radius of the lens.

Finally, we discuss implications of our results for LISA. We take the SMBHs mergers as the sources for which the signal-to-noise ratio $(\mathrm{SNR})$ is very high, $\mathrm{SNR} \sim 10^{3}$. In the matched filtering analysis, the waveform (the amplitude and the phase) can be measured to an accuracy approximately equal to $(\mathrm{SNR})^{-1} \sim 0.1 \%$ [1]. But, the ratio $s / r$ (the lens size to the distance) is much smaller than this value for lensing by galaxies or galaxy clusters. 

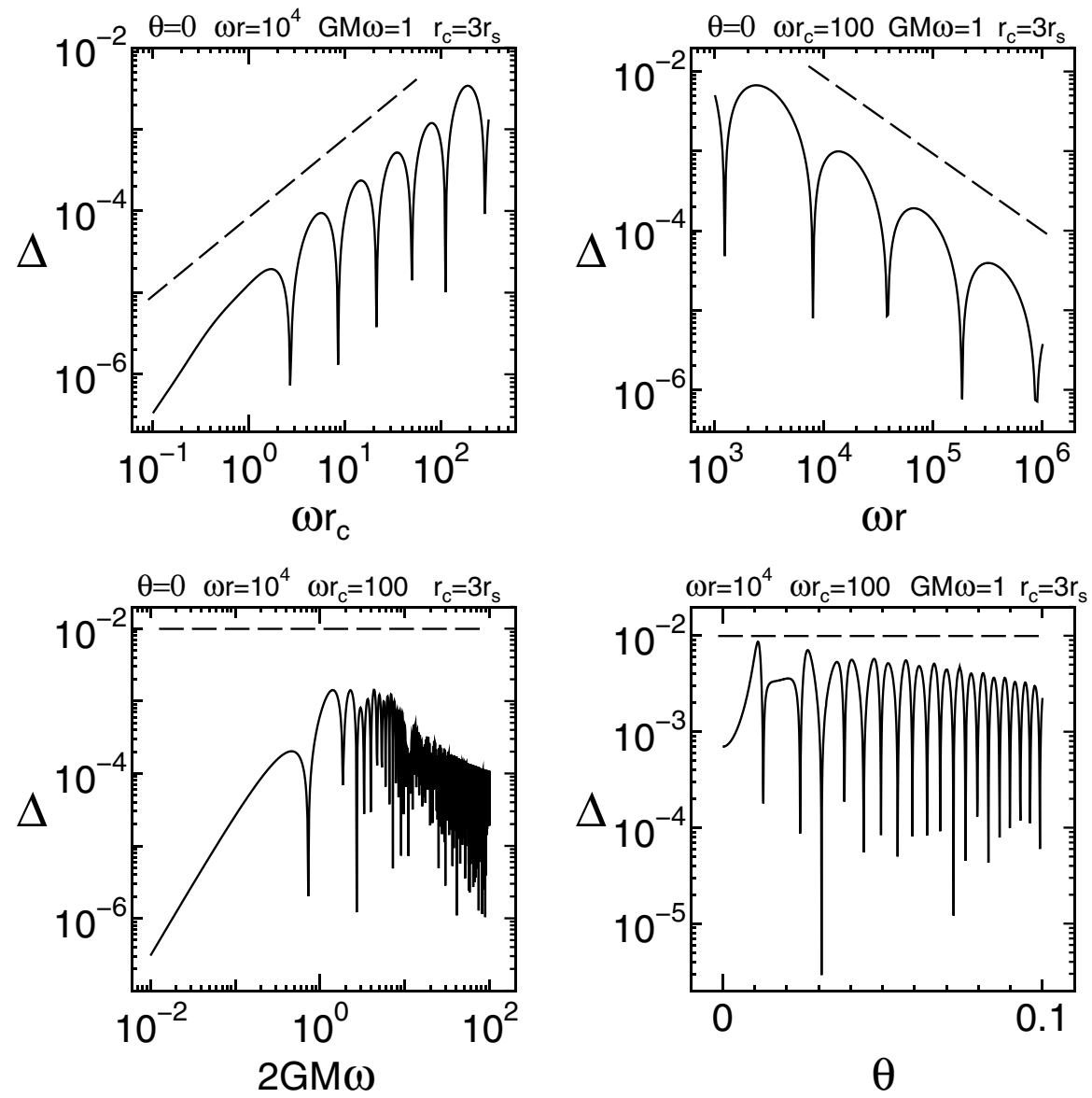

FIG. 5. Same as Fig. 3, but for the Hernquist model. The dashed lines denote the ratio of cutoff radius to distance $r_{c} / r$.

Hence the thickness of lens cannot be measured and the thin-lens approximation is valid in real observational situation. If lensed signal is detected with high SNR, the three lens models can be distinguished from lensing observables such as magnification, time delay, and image numbers.

\section{ACKNOWLEDGMENTS}

We would like to thank Takahiro Tanaka for useful comments and encouragement. We would also like to thank Takashi Nakamura, Misao Sasaki, Atsushi Taruya, and Makoto Yoshikawa for useful comments.
[1] C. Cutler and E. E. Flanagan, Phys. Rev. D 49, 2658 (1994).

[2] H. C. Ohanian, Int. J. Theor. Phys. 9, 425 (1974); H. C. Ohanian, Astrophys. J. 271, 551 ( 1983).

[3] P. V. Bliokh and A. A. Minakov, Astrophys. Space Sci. 34, L7 (1975).

[4] R. J. Bontz and M. P. Haugan, Astrophys. Space Sci. 78, 199 (1981)

[5] K.S. Thorne, Gravitational Radiation, edited by N. Deruell and T. Piran (North-Holland, Amsterdam, 1983), p. 28.

[6] S. Deguchi and W. D. Watson, Astrophys. J. 307, 30 (1986).

[7] http://lisa.jpl.nasa.gov.
[8] M. G. Haehnelt, Mon. Not. R. Astron. Soc. 269, 199 (1994).

[9] R. Takahashi and T. Nakamura, Astrophys. J. 595, 1039 (2003).

[10] N. Seto, Phys. Rev. D 69, 022002 (2004).

[11] K. Yamamoto and K. Tsunoda, Phys. Rev. D 68, 041302 (2003).

[12] F. de Paolis, G. Ingross, A. A. Nucita, and A. Qadir, Astron. Astrophys. 394, 749 (2002).

[13] T. T. Nakamura, Phys. Rev. Lett. 80, 1138 (1998).

[14] K. Yamamoto, astro-ph/0309696.

[15] T. T. Nakamura and S. Deguchi, Prog. Theor. Phys. Suppl. 133, 137 (1999).

[16] C. Baraldo, A. Hosoya, and T. T. Nakamura, Phys. Rev. D 59, 083001 (1999). 
[17] P. Schneider, J. Ehlers, and E. E. Falco, Gravitational Lenses (Springer, New York, 1992).

[18] P. C. Peters, Phys. Rev. D 9, 2207 (1974).

[19] N. Andersson and B. P. Jensen, gr-qc/0011025.

[20] M. Abramowitz and I. A. Stegun, Handbook of Mathematical Functions (Dover, New York, 1970).
[21] A. Messiah, Quantum Mechanics (Intersicence, New York, 1961).

[22] L. Hernquist, Astrophys. J. 356, 359 (1990); C. R. Keeton, astro-ph/0102341. 